\title{
Multiple Antenna Systems With Finite Rate Feedback
}

\author{
Chandra R. Murthy, Jun Zheng and Bhaskar D. Rao \\ Department of Electrical and Computer Engineering \\ University of California, San Diego \\ La Jolla, CA 92093-0407 \\ Email: \{cmurthy, juzheng, brao\}@ucsd.edu
}

\begin{abstract}
The performance of multiple antenna systems can be greatly improved by making the channel state information (CSI) available at the transmitter. In practice, therefore, a finite-rate feedback link is employed to convey a quantized version of the CSI to the transmitter. This semi-tutorial paper summarizes some of the recent work on the design and analysis of multiple antenna systems with finite rate feedback. In particular, we discuss appropriate criteria for the design of quantizers for feedback-based communication systems along with corresponding algorithms for codebook generation. Finally, we qualitatively show how the classical source coding theory can be extended to analyze the performance of finite rate feedbackbased multiple antenna systems.
\end{abstract}

\section{Introduction ANd Problem Setup}

Multiple antenna systems have received much attention for the past decade or so, due to their promise of higher data rates compared to their single-antenna counterparts. When the multiple antenna system comprises of $t$ transmit and $r$ receive antennas, if we consider the channel to be flat-fading, the input-output relation can be expressed as

$$
\mathbf{y}=H \mathbf{x}+\mathbf{n},
$$

where $\mathbf{x} \in \mathbb{C}^{t}$ is the channel input, $\mathbf{y} \in \mathbb{C}^{r}$ is the channel output, $\mathbf{n} \in \mathbb{C}^{r}$ is the AWGN at the receiver, and $H \in \mathbb{C}^{r \times t}$ is the channel matrix with the $(i, j)$-th entry containing the complex gain from the $j$-th transmit antenna to the $i$-th receive antenna. Under the block fading model, the channel matrix $H$ remains constant within a fading block, and changes independently from block to block according to some statistical distribution (e.g., Rayleigh distribution). In this case, we can omit the time index in writing the input-output relation, as

This work was supported in part by UC Discovery grant nos. Com 02-10105, Com 02-10109, Conexant Systems and Ericsson, and in part by, the U. S. Army Research Office under the Multi-University Research Initiative (MURI) grant \# W911NF-04-1-0224. we have done above. It is well known that the performance and complexity of multiple-input multipleoutput (MIMO) systems can be improved by making the channel state information (CSI) available at the transmitter. In general, the CSI has to be conveyed back to the transmitter from the receiver through a feedback link. However, in practical communication systems, the CSI feedback suffers from impairments such as channel estimation errors, quantization errors, feedback delay and feedback errors. Recently, several interesting papers have appeared, quantifying the effects of the partial CSI at the transmitter caused by these impairments. Channel feedback is also under consideration in 3rd generational mobile and wireless LAN standards, for example in the closed-loop mode specification in 3GPP High Speed Downlink Packet Access (HSDPA) [1] and in the eigenbeamforming mode specification in IEEE 802.11 [2] and IEEE 802.16 [3].

Two of the popular models for studying the effect of partial CSI at the transmitter are statistical feedback and instantaneous feedback. In the statistical feedback approach, it is assumed that the channel coherence time is too small to feedback every channel instantiation. However, the channel statistics vary sufficiently slowly, so that the mean and/or the covariance of the channel can be made available to the transmitter accurately. The channel is then modelled as Gaussian distributed with the given mean and covariance, and the system performance is optimized with respect to the input distribution and analytically characterized. Examples of works that employ statistical feedback include [4] - [10].

In the instantaneous feedback approach, which is the focus of this paper, the receiver attempts to convey to the transmitter the current channel instantiation, typically through a bandwidth-constrained feedback link. That is, given $B$ bits of feedback, the receiver maps the current channel instantiation $H$ to one of $N=2^{B}$ integer indices, with each index corresponding to a particular 
mode of the channel. The transmitter has knowledge of the $N$-mode codebook, and therefore, it is able to optimize its transmission strategy based on the feedback information. Thus, it is a very interesting and challenging problem to design optimal quantization schemes and the associated transmission strategies for multiple-antenna systems with finite rate feedback. The design and analysis of the optimum quantizer that takes advantage of both the underlying channel distribution as well as the performance metric (received SNR, outage probability, mutual information rate, bit error rate, etc) has received much attention in the past few years, notably in [11] [24]. A recent overview of work in this area is [25].

There appear to be similarities between classical fixedrate source coding and channel quantization. For example, in the source coding problem, the encoder attempts to describe a random source by a finite number of bits, and the goal is to minimize the representation error (for example, the Euclidean norm of the quantization error). In channel quantization, one would like to describe the channel state by a finite number of bits, and the goal is to optimize a given performance metric (such as the received SNR, channel throughput or BER). Although several authors have remarked on this similarity (including [11], [14] [26]), the exact connection between the two fields has remained elusive. In this paper, we will present qualitative arguments to show how to bridge the gap between these two fields. This paper is organized as follows. In section II, we present a summary of some of the recent work in the area of quantized feedback based systems. In section III, we present the key approach used in classical source coding literature and examine the similarities and differences between source coding and channel quantization. We show how the quantizer design problem is in fact a more general source compression problem. We briefly summarize some of our recent findings in this area as well.

\section{REVIEW OF PAST WORK}

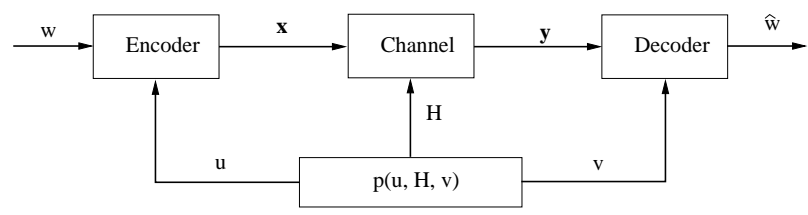

Fig. 1. Model of a typical multiple antenna system with finite rate instantaneous feedback.

In this section, we will present a review of the recent work in the area of multiple antenna system design with quantized feedback. We illustrate a general model of a multiple antenna system with finite rate instantaneous feedback in Fig. 1. The channel state information (CSI) $H$, CSI at the receiver (CSIR) $v$, and CSI at the transmitter (CSIT) $u$ follow a joint distribution given by $p(u, H, v)$. Note that this model includes situations where the CSIR is perfect $(v=H)$, as well as situations where the CSIR and CSIT suffer from estimation error or feedback errors ( $u, v$ and $H$ are correlated).

Skoglund et al [9] investigated the system in Fig. 1 from an information theoretic perspective. They proved that when the receiver has full knowledge of the CSI and the feedback information, i.e. $v=(\mathbf{H}, u)$, the capacity-achieving encoder subject to a total transmit power constraint can be split into two parts, a fixed codebook encoder and an adaptive weighting matrix $\mathbf{W}(u)$ based on the feedback information $u$. The optimal input distribution of the fixed encoder is spatially white i.i.d. complex Gaussian.

Lau et al [19] studied a more general finite rate feedback system where the channel state $s=\mathbf{H}$, the CSIR $v$, and the CSIT $u$ have a joint statistical relation $p(s, u, v)$. They proved that the optimal quantizer is a time-invariant deterministic function. The system capacity is obtained through a double optimization over both the quantization function and the input distribution $p_{\mathbf{x} \mid u}\left(\mathbf{x} \mid u_{q}\right)$ conditioned on the feedback information $u_{q}$, with $1 \leq q \leq N$. However, this joint optimization problem is difficult to solve, and numerical techniques have to be used to provide approximate solutions. Hence, most of the attention in recent literature is focussed on the case of ideal CSIR, and simple sub-optimal input distributions (i.i.d Gaussian distribution) or suboptimal transmission schemes (such as employing just a single beamforming vector or a pre-coding matrix with orthonormal column vectors).

In [11], Narula et al considered a MISO system with perfect CSIR and employed the finite-rate feedback link to describe a beamforming vector. Both the channel gain as well as the mutual information were used as performance metrics. The Lloyd algorithm [27] for vector quantization (VQ) was utilized for designing the optimal codebook of beamforming vectors. On the analytical side, the authors took advantage of the connection between the quantization problem and the classical rate distortion theory. They derived an expression for the SNR loss due to quantized beamforming as a function of the number of feedback bits $B$ as

$$
\rho_{L}=\rho_{P}-\rho_{Q} \simeq \frac{t-1}{t} 2^{\frac{-B}{t}},
$$

where $\rho_{P}$ is the expected received SNR with perfect beamforming and $\rho_{Q}$ is the expected received SNR with 
quantized beamforming. The above result is based on the assumption that the number of transmit antennas $t$ is large.

Mukkavilli et al [14] were able to derive a lower bound on the outage probability of quantized beamforming for MISO systems with i.i.d. Rayleigh fading channels. They employed a geometrical approach to derive the fractional loss in outage $F(B)$, defined as the relative loss in outage performance between the best beamformer with $B$ bits of feedback when compared to a beamformer with perfect CSI at the transmitter, for large SNR. They obtained an expression of the form

$$
F(B)=(t-1) 2^{\frac{-B}{t-1}} .
$$

This result is suitable for an arbitrary number of transmit antennas $t$, and the analysis is based on the geometrical properties of the channel space. The authors also considered the codebook design for the quantized beamforming vectors, and proposed a min-max criterion based on minimizing the maximum inner product between any two distinct beamforming vectors in the codebook. The outage probability of the codebook designed based on this criterion was shown to be very close to the lower bound for small $t$ and $B$.

Love et al [15], [16], [24] also proposed the above min-max criterion for designing the codebook in a i.i.d. Rayleigh fading MIMO channel setting, and related the problem to that of Grassmannian line packing [28], which is the problem of maximally separating lines in the Grassmann manifold. A random computer searching algorithm was employed to generate a codebook that optimizes the Grassmannian beamforming criterion. They provided necessary and sufficient conditions for the quantized feedback scheme to achieve full diversity order. They also obtained bounds on the codebook size to satisfy a given loss in SNR or capacity by using results on Grassmannian line packing such as the Rankin bound. Another problem the authors have addressed is quantized equal gain transmission [29], i.e., transmit beamforming under a per-antenna power constraint. They showed that the Grassmannian beamforming criterion can still be used to obtain good beamforming vector codebooks.

Vector quantization (VQ) techniques along with the Lloyd algorithm can be used to obtain codebooks that specifically account for both the statistical distribution of the channel as well as the performance metric (for example, the mutual information rate or the average received SNR). This approach was used by Xia et al in [21], [22], [26] to derive a criterion for designing the quantized beamforming codebook in a MISO channel to maximize the average received $\mathrm{SNR}$ as

$$
\max _{\mathcal{Q}(\cdot)}\left[\left|\mathbf{h}^{H} \mathcal{Q}(\mathbf{h})\right|^{2}\right]
$$

where $\hat{\mathbf{v}}=\mathcal{Q}(\mathbf{h})$ is the quantized beamforming vector $(\|\hat{\mathbf{v}}\|=1)$ corresponding to the channel instantiation h. Simultaneously, Roh et al [18], [30] considered the system capacity loss as their performance metric. The capacity loss was defined as the difference in expected system mutual information rate between perfect beamforming (i.e., with infinite feedback rate) and quantized beamforming. They derived a weighted inner product criterion for the codebook design in a MISO setting as follows:

$$
\max _{\mathcal{Q}(\cdot)}\left[\left(\frac{\|\mathbf{h}\|^{2} P_{T}}{1+\|\mathbf{h}\|^{2} P_{T}}\right)\left|\mathbf{v}^{H} \mathcal{Q}(\mathbf{h})\right|^{2}\right],
$$

where $\mathbf{v} \triangleq \mathbf{h} /\|\mathbf{h}\|$. The Lloyd algorithm [27], which is a standard algorithm in VQ literature, was used to generate the codebook. The algorithm consists of two iterative computing steps: the nearest neighborhood condition and the centroid condition. At the end of each iteration, the objective function (performance metric) always improves, thus guaranteeing the convergence of the algorithm to a (local) optimum. Both of these groups analyzed the performance of MISO systems with limited rate-feedback in the case of i.i.d. Rayleigh fading channels, and obtained an expression of the capacity loss (or SNR loss) as

$$
C_{\text {Loss }}=\frac{t-1}{t} 2^{-\frac{B}{t-1}} .
$$

The power of these vector quantization based techniques is that at high rates, they can be used to design codebooks that are optimal for any given distortion function (such as SNR loss, capacity loss, BER, etc) and any given channel distribution.

In [31], Roh et al extended the results from MISO studies to the case of MIMO systems with quantized feedback. They employed a transmission scheme with a variable number of spatial channels, where the total available power is allocated equally to the spatial channels. They proposed a new criterion for designing the codebook of beamforming matrices based on minimizing the system mutual information loss resulting from the limited rate in the feedback channel. Using the criterion, a similar iterative Lloyd-type design algorithm was developed that converges to an optimum codebook. By utilizing the complex multivariate beta distribution and tractable approximations to the Voronoi regions associated with each code point, the effect on the system mutual information rate of the finite-bit representation 
of the beamforming matrix was analyzed for the case of i.i.d. Rayleigh fading channel in the high SNR regime. Furthermore, to compensate for the degradation due to the equal power allocation assumption, a multi-mode spatial multiplexing transmission strategy was proposed, wherein the number of data streams is determined based on the average SNR.

Scalar quantization of the MIMO channel parameters in slowly-time varying channels was considered in [32], where the authors used Givens rotations to parameterize the eigenvectors of the covariance matrix that is employed at the transmitter. Since the parameters are smoothly changing in time for slowly time-varying channels, adaptive delta modulation was employed to quantize and feedback each parameter. The proposed feedback scheme is able to track the time-varying channel and achieves a capacity close to the perfect feedback case with a reasonable feedback rate. In [33], the authors considered quantization of the unitary MIMO precoding matrix, and proposed a matrix factorization approach to exploit the spatial structure inherent in the unitary matrix. In [34], the authors employed a vector quantization based approach for per-antenna power constrained beamforming. They derived a modified Lloyd algorithm to design the codebook with an approximation capacity loss as the performance metric, and also obtained analytical expressions for the capacity loss with quantized feedback.

\section{SOURCE CODING VERSus CHANNEL QUANTIZATION: SimilaritiES AND DIFFERENCES}

In classical source coding, the encoder describes a random source $\mathbf{s} \in \mathbb{R}^{k}$ by one of the entries in a codebook denoted $\left\{\hat{\mathbf{s}}_{1}, \ldots, \hat{\mathbf{s}}_{N}\right\}$, where $\hat{\mathbf{s}}_{i} \in \mathbb{R}^{k}$. The goal of the encoder is to minimize the distortion (for example, the expected $r$-th power Euclidean distance) between $\mathbf{s}$ and its quantized version $\hat{\mathbf{s}}_{i}$. Source coding is a well established field, and a vast body of results are available for both the design and analysis of optimal quantizers (see [35] and the references therein, for a comprehensive survey of source quantization). Hence, if the exact relationship between source coding and channel quantization were established, one could draw from source coding results to design as well as analyze the performance of feedback based systems.

The major differences between channel quantization and the classical source coding are:

1) Not all channel parameters need to be quantized. For example, consider the quantization of the maximum ratio transmission (MRT) beamforming vector in a MISO system, which is given by $\mathbf{v} \triangleq$ $\mathbf{h} /\|\mathbf{h}\|[36]$. We are only interested in the direction of the channel vector $\mathbf{h}$ rather than in its gain $\|\mathbf{h}\|$. Clearly, it is redundant to directly quantize the channel instantiation $\mathbf{h}$.

2) The channel instantiation and the actual variable to be quantized may lie in different spaces and may have different dimensions. In the example of quantized MRT beamforming, the vector $\mathbf{v} \in \mathbb{C}^{t}$ that we need to quantize is constrained to be unit-norm and hence lies on the unit hyper-sphere or manifold, whereas the channel instantiation $\mathbf{h}$ could be anywhere in the $\mathbb{C}^{t}$ space.

3) The additional information which is not the quantization objective, for example the gain $\|\mathbf{h}\|$ of the MISO channel, can be utilized as side information at the quantizer (or the receiver) to improve the quantization performance.

4) The distortion measure may be a more general non-mean-square error function, for example, if the average received SNR loss is the performance metric, then the distortion measure is given by

$$
\rho_{L} \triangleq \mathbf{E}\left\{P_{T} \cdot\|\mathbf{h}\|^{2} \cdot\left(1-\left|\mathbf{v}^{H} \hat{\mathbf{v}}\right|^{2}\right)\right\},
$$

where $P_{T}$ is the transmit power (assuming the AWGN at the receiver has unit variance). Notice that the distortion function is clearly not of the form $\|\mathbf{v}-\hat{\mathbf{v}}\|^{r}$, i.e., not a distance distortion function.

Due to the above reasons, classical rate distortion results cannot be directly applied to the design and analysis of finite rate feedback systems. In order to take advantage of the vast body of literature on source coding, we need to modify the source coding problem to allow for constrained quantization and non-meansquared distortion functions. We start by summarizing some of the major approaches used in classical source coding studies and show how it may be extended to fit our case of interest.

In 1948, W. R. Bennett [37] used a companding model for nonuniform scalar quantization and showed that the distortion could be approximated by the integral

$$
D \simeq \frac{1}{12 N^{2}} \int p(x)\left[E^{\prime}(x)\right]^{-2} d x,
$$

for the mean-squared quantizing error where $N$ is the number of levels, $p(x)$ is the probability density of the source symbols, and $E^{\prime}(x)$ is the slope of what he called the compressor curve. The problem was revisited by Gersho in [38], where he solved the problem for the more general case of $k$-dimensional vector quantization with the $r$-th power Euclidean distance distortion function. He 
obtained the rate-distortion formula

$$
D \simeq C(k, n) N^{-r / k}\|p(x)\|_{k /(k+r)},
$$

where $C(k, n)$ is a constant known as the moment of inertia coefficient, and $\|p(x)\|_{\alpha} \triangleq\left[\int[p(x)]^{\alpha} d x\right]^{1 / \alpha}$. This equation provides critical insight into the asymptotic distortion analysis and forms the basis of most of the later work in the area of source coding. Two extensions of the asymptotic distortion analysis were provided by $\mathrm{Na}$ and Neuhoff in [39] and by Gardner and Rao in [40]. Na and Neuhoff [39] showed how the loss in performance of a (possibly sub-optimal) quantizer relative to the optimum quantizer can be decomposed into the point density loss and the cell-shape loss. The point density loss arises because the optimum quantizer places the quantizated vectors differently compared to (say) a structured codebook, whereas the cell-shape loss arises because the partition region differs from the optimum Voronoi shape. Gardner and Rao [40] considered an arbitrary (a general non-mean-squared form) distortion function and derived an asymptotic analysis based on a second order approximation of the distortion function. They showed that the distortion approaches a simple quadratically weighted error measure, where the weighting matrix (called the sensitivity matrix) captures the scalar sensitivities of the individual parameters as well as the cross-sensitivity terms arising from the interaction in quantizing multiple parameters simultaneously.

\section{BRIdGing the Gap Between Source Coding And Finite Rate FEEDbaCk Systems}

In this section, we qualitatively describe the machinery needed to bridge the gap between source coding and finite rate feedback systems. Specifically, the four major differences alluded to in the previous section are addressed in the following.

1) Since not all the information in the CSI needs to be quanitzed, the first step is to parameterize the channel information $\mathbf{h}=\left[h_{1}, \ldots, h_{t}\right]^{T}$ as $(\mathbf{y}, \mathbf{z})$, where $\mathbf{y}$ contains the smallest subset of free-parameters that need to be quantized and $\mathbf{z}$ is all the remaining parameters that serve as additional information for the quantizer at the receiver. In the case of quantized MRT beamforming in MISO systems, for example, $\mathbf{y}$ consists of the $2(t-1)$ parameters that describe the relative phase and relative amplitude of $h_{2}, \ldots, h_{t}$ with respect to $h_{1}$. Also, $\mathbf{z}$ consists of $h_{1}$, i.e., the two parameters describing its amplitude and phase.

The key step is to quantize only the minimum set of parameters and to ensure that the quantizer makes appropriate use of the side information $\mathbf{z}$. It turns out that the optimal vector quantizer will always have the same performance although the parametrization is not unique and different parameterizations will lead to different quantization algorithms and codebooks. The choice of the parametrization is important from the point of view of designing the codebook and encoder, since some parameterizations yield simpler designs than others.

2) Since the channel and the quantized vector lie in different spaces, Gersho's asymptotic analysis [38] cannot be directly employed in the channel space. Instead, it should be applied in the reduced-dimension parameter space $\mathbf{y}$, accounting for any constraints on the parameters.

3) The additional information in the parameters $\mathbf{z}$ at the encoder does not have to be quantized, however, it can be used to improve the performance of the encoder. The encoder thus has to be designed to take this side information into account. As a simple example, consider the case of correlated fading MISO channel with quantized MRT beamforming, the gain information $\|\mathbf{h}\|$ is not statistically independent of the direction $\mathbf{v}$. If the distortion is given by (7), it is clear that the optimum encoder must try to quantize the beamforming vectors $\mathbf{v}$ corresponding to channels with larger $\|\mathbf{h}\|$ more accurately than those with smaller $\|\mathbf{h}\|$ since the distortion is proportional to $\|\mathbf{h}\|^{2}$.

4) The case of non-mean squared distortion function has already been considered in [40]. Two important differences from the past approach is that (a) we need to use a projected sensitivity matrix, where the conventional sensitivity matrix is projected onto a space that is orthogonal to the gradient of the constraint conditions; and (b) the distortion function is parameterized by the additional information $\mathbf{z}$ (see item (3) above).

Due to space considerations, we have only provided a brief description of how to extend the asymptotic analysis of source coding to the case of finite rate feedback systems. Interestingly, after detailed derivations, it can be proved [41] that the high-SNR, high-resolution (large $B$ ) performance of MISO systems with quantized MRT beamforming is in fact given by (6), though it was obtained from a completely different perspective. Furthermore, the source coding framework is sufficiently general to include many more complicated finite rate feedback systems. As an example, consider the case of i.i.d. Rayleigh fading MIMO channel with $t$ transmit and $r$ receive antennas and using $n$ beamforming vectors (as a pre-coding matrix) with equal power allocation. The system distortion function is the average mutual information loss, i.e., the the difference between the average mutual information with perfect pre-coding and 
that of finite-rate quantized pre-coding. It can be shown that, in the high-SNR and high-resolution regime, the mutual information loss $I_{\text {Loss }}$ is tightly lower bounded by the following form

$$
\begin{aligned}
I_{\text {Loss }} \geq & \left(\frac{[(t-n) n] !(n-1) ! !(t-n-1) ! !}{(t-1) ! !}\right)^{1 / n} \\
& \times \frac{(t-n) n}{(t-n) n+1} 2^{-\frac{B}{(t-n) n}}
\end{aligned}
$$

where we define $k ! ! \triangleq k !(k-1) ! \cdots 1$ !.

\section{Conclusions}

In this paper, we have summarized some of the recent work on the design and analysis of multiple antenna systems with finite rate feedback. In particular, we have discussed appropriate criteria for the design of quantizers for feedback-based communication systems and corresponding algorithms for codebook generation. We have also summarized the source-coding problem and explained how it is similar and different from the quantization problem for channels with finite rate feedback, and provided qualitative arguments to show how to deal with the differences. Our hope is that this can be used to glean useful insights into the design and analysis of finite rate feedback-based communication systems by drawing on the vast body of source coding literature that is available.

\section{REFERENCES}

[1] 3GPP TS25.308, "UTRA high speed downlink packet access (HSDPA); overall description; stage 2," version 5.5.0.

[2] IEEE P802.11, "TGn sync proposal technical specification," doc.: IEEE 802.11-04/0889r4.

[3] IEEE P802.16, "IEEE standard for local and metropolitan area networks part 16: Air interface for fixed broadband wireless access systems," IEEE Std 802.16-2004.

[4] S. A. Jafar, S. Vishwanath, and A. Goldsmith, "Channel capacity and beamforming for multiple transmit and receive antennas with covariance feedback," in Proc. IEEE ICC 2001, pp. 2266 -2270 , June 2001

[5] E. Visotsky and U. Madhow, "Space-time transmit precoding with imperfect feedback," IEEE Trans. Inform. Theory, vol. 47, pp. 2632-2639, Sept. 2001.

[6] E. G. Larsson, G. Ganesan, P. Stoica, and W.-H. Wong, "On the performance of orthogonal space-time block coding with quantized feedback," IEEE Commun. Letters, vol. 6, pp. 487489, Nov. 2002.

[7] S. Zhou and G. B. Giannakis, "Adaptive modulation for multiantenna transmissions with channel mean feedback," IEEE Trans. Wireless Commun., vol. 3, pp. 1626-1636, Sept. 2004.

[8] A. L. Moustakas and S. H. Simon, "Optimizing multiantenna systems with partial channel knowledge," in Proc. Seventh Int. Symposium on Sig. Proc. and Its Applications, vol. 1, pp. $217-$ 220, July 2003.
[9] M. Skoglund and G. Jongren, "On the capacity of a multipleantenna communication link with channel side information," IEEE J. on Select. Areas in Commun., vol. 21, pp. 395-405, Apr. 2003.

[10] E. A. Jorsweick and H. Boche, "Optimal transmission strategies and impact of correlation in multiantenna systems with different types of channel state information," IEEE Trans. on Sig. Proc., vol. 52, pp. 3440-3453, Dec. 2004.

[11] A. Narula, M. J. Lopez, M. D. Trott, and G. W. Wornell, "Efficient use of side information in multiple-antenna data transmission over fading channels," IEEE J. Select. Areas Commun., vol. 16, pp. 1423 - 1436, Oct. 1998.

[12] S. Bhashyam, A. Sabharwal, and B. Aazhang, "Feedback gain in multiple antenna systems," IEEE Trans. on Commun., vol. 50, pp. 785-798, May 2002.

[13] R. S. Blum, "MIMO with limited feedback of channel state information," in Proc. Int. Conf. on Acoustics, Speech, and Signal Proc. (ICASSP), vol. 4, pp. 89-92, Apr. 2003.

[14] K. K. Mukkavilli, A. Sabharwal, E. Erkip, and B. Aazhang, "On beamforming with finite rate feedback in multiple-antenna systems," IEEE Trans. Inform. Theory, vol. 49, pp. 2562-2579, Oct. 2003.

[15] D. J. Love, R. W. Heath, Jr., and T. Strohmer, "Quantized maximal ratio transmission for multiple-input multiple-output wireless systems," in Proc. Asilomar Conf. 2002, (Pacific Grove, CA), Nov. 2002.

[16] D. J. Love, R. W. Heath, Jr., and T. Strohmer, "Grassmannian beamforming for multiple-input multiple-output wireless systems," IEEE Trans. Info. Theory, vol. 49, pp. 2735-2747, Oct. 2003.

[17] W. Santipach and M. L. Honig, "Asymptotic performance of MIMO wireless channels with limited feedback," in Proc. MILCOM, (Boston, MA), pp. 141-146, Oct. 2003.

[18] J. C. Roh and B. D. Rao, "Performance analysis of multiple antenna systems with VQ-based feedback," in Proc. Asilomar Conf. 2004, (Pacific Grove, CA), Nov. 2004.

[19] K. N. Lau, Y. Liu, and T. A. Chen, "On the design of MIMO block-fading channels with feedback-link capacity constraint," IEEE Trans. Commn., vol. 52, pp. 62-70, Jan. 2004.

[20] J. Choi and R. W. Heath, Jr., "Interpolation based transmit beamforming for MIMO-OFDM with limited feedback," in Proc. IEEE ICC, vol. 1, pp. 249 - 253, June 2004.

[21] P. Xia, S. Zhou, and G. B. Giannakis, "Multiantenna adaptive modulation with beamforming based on bandwidth- constrained feedback," IEEE Trans. on Commn., vol. 53, pp. 526-536, Mar. 2005.

[22] P. Xia and G. B. Giannakis, "Design and analysis of transmitbeamforming based on limited-rate feedback," in Proc. IEEE VTC, Sept. 2004.

[23] G. Jongren and M. Skoglund, "Quantized feedback information in orthogonal space-time block coding," IEEE Trans. Inform. Theory, vol. 50, pp. 2473-2486, Oct. 2004.

[24] D. J. Love and R. W. Heath, Jr., "Limited feedback unitary precoding for orthogonal space-time block codes," IEEE Trans. on Sig. Proc., vol. 53, pp. 64-73, Jan. 2005.

[25] D. J. Love, R. W. Heath, Jr., W. Santipach, and M. L. Honig, "What is the value of limited feedback for mimo channels?," IEEE Comm. Mag., vol. 42, pp. 54-59, Oct. 2004.

[26] P. Xia and G. B. Giannakis, "Design and analysis of transmitbeamforming based on limited-rate feedback," IEEE Trans. on Sig. Proc., 2005 (to appear).

[27] A. Gersho and R. M. Gray, Vector Quantization and Signal Compression. Kluwer Academic, 1992.

[28] J. H. Conway, R. H. Hardin, and N. J. A. Sloane, "Packing lines, 
planes, etc.: Packings in Grassmannian space," Experimental Math., no. 5, pp. 139 - 159, 1996.

[29] D. J. Love and R. W. Heath, Jr., "Equal gain transmission in multiple-input multiple-output wireless systems," IEEE Trans. Commn., vol. 51, pp. 1102-1110, July 2003.

[30] J. C. Roh, Multiple-Antenna Communication with Finite Rate Feedback. PhD thesis, Univ. of California, San Diego, 2005.

[31] J. C. Roh and B. D. Rao, "MIMO spatial multiplexing systems with limited feedback," in Proc. IEEE ICC 2005, (Seoul, Korea), May 2005.

[32] J. C. Roh and B. D. Rao, "An efficient feedback method for MIMO systems with slowly time-varying channels," in IEEE WCNC 2004, (Atlanta, Georgia, USA), Mar. 2004.

[33] J. C. Roh and B. D. Rao, "Channel feedback quantization methods for MISO and MIMO systems," in IEEE International symposium on Personal, Indoor and Mobile Radio Communications (PIMRC) 2004, (Barcelona, Spain), Sept. 2004.

[34] C. R. Murthy and B. D. Rao, "A vector quantization based approach for equal gain transmission," in Proc. Globecom, (St. Louis, MO), Nov. 2005.

[35] R. M. Gray and D. L. Neuhoff, "Quantization," IEEE Trans. Info. Theory, vol. 44, no. 6, pp. 2325-2383, 1998.

[36] T. K. Y. Lo, "Maximum ratio transmission," IEEE Trans. Commun., vol. 47, pp. 1458-1461, Oct. 1999.

[37] W. R. Bennett, "Spectra of quantized signals," Bell Syst. Tech. Journal, vol. 27, pp. 446 - 472, 1948.

[38] A. Gersho, "Asymptotically optimum block quantization," IEEE Trans. Info. Theory, vol. IT-25, no. 4, pp. 373-380, 1979.

[39] S. Na and D. Neuhoff, "Bennett's integral for vector quantizers," IEEE Trans. Inform. Theory, vol. 41, no. 4, pp. 886 - 900, 1995.

[40] W. R. Gardner and B. D. Rao, "Theoretical analysis of highrate vector quantization of LPC parameters," IEEE Trans. on Speech and Audio Proc., vol. 3, pp. 367-381, Sept. 1995.

[41] J. Zheng and B. D. Rao, "Multiple antenna systems with finite rate feedback: Part I : Asymptotic analysis from a source coding perspective," Submitted to IEEE Trans. Sig. Proc. 